\title{
Upstream Flow Control for the Savonius Rotor under Various Operation Conditions
}

\author{
Can Kang ${ }^{1, * \mathbb{D}}$, Wisdom Opare ${ }^{1,2}$, Chen Pan ${ }^{1}$ and Ziwen Zou ${ }^{1}$ \\ 1 School of Energy and Power Engineering, Jiangsu University, Zhenjiang 212013, China; \\ 5103171309@stmail.ujs.edu.cn (W.O.); lynnpc93@163.com (C.P.); zouziwenjs@126.com (Z.Z.) \\ 2 Faculty of Engineering, Takoradi Technical University, Takoradi P.O. Box 256, Ghana \\ * Correspondence: kangcan@ujs.edu.cn; Tel.: +86-511-88780217
}

Received: 30 April 2018; Accepted: 1 June 2018; Published: 6 June 2018

\begin{abstract}
Applications of the Savonius rotor have been extended in recent years, necessitating an in-depth investigation on flow characteristics of such a fluid energy converting device. For the wake flow downstream of the Savonius rotor, studies have been reported extensively. Nevertheless, literature specifically devoted to the upstream flow of the Savonius rotor can rarely be found. This review collects and compiles findings from relevant studies to prove the significance of upstream flow patterns to the operation of the Savonius rotor. Then attempts from experimental and numerical aspects to substantiate the important effect of the upstream flow are implemented. Based on practical cases and laboratory works, upstream flow patterns for the Savonius rotor are divided into four types, namely uniform flow, guided flow, rotor wake flow and oscillating flow. Accordingly, conditions under which these upstream flow patterns arise are analyzed respectively. Experimental and numerical results are presented to clarify the influential factors underlying diverse upstream flow patterns. Furthermore, the relationship between the performance of the Savonius and the upstream flow is elucidated, facilitating the development of techniques of controlling the upstream flow. This review provides a systematic reference for the control of the upstream flow for the Savonius rotor, which has the tendency of developing into an independent technical branch.
\end{abstract}

Keywords: Savonius rotor; upstream flow; auxiliary device; multiple rotors; experimental condition; rotor performance

\section{Introduction}

The Savonius rotor is the representative of the drag-type wind rotors and has attracted much attention since its debut [1]. The advantage of the Savonius rotor is evident as it is applied to pump water from the well in rural areas or to generate electricity for household use [2]. In recent years, various Savonius rotors have been reported [3]. The Savonius rotor plays dual roles: one is as an energy converter and the other is as the solid boundary that dominates the surrounding flow characteristics. Meanwhile, as the Savonius rotor rotates, a periodic disturbance will be imposed on the ambient fluid; thus flow patterns and rotor performance change inter-relatedly with rotor rotation.

In terms of fluid flow in the vicinity of the Savonius rotor, the wake flow has been studied extensively [4]. In contrast, specific treatment of the upstream flow has rarely been reported. Essentially, the rotor performance depends largely on the upstream flow pattern. Measures of controlling the upstream flow such as deploying auxiliary device like the curtain or the deflector have been attempted [5]. In this aspect, both flow characteristics and technical issues have to be considered [6]. For instance, the intrusion of the auxiliary device might produce considerably different upstream flow relative to the original one. Another example is to control the upstream flow velocity through adjusting the car speed during the tow testing [7]. Intricate upstream flows are often encountered in practical applications of the 
Savonius rotor, as is appreciable from the cases featured by the integration of the Savonius rotor with buildings [8]. Apart from frequently varied wind direction, the upstream flow is exposed to wall effects of the adjacent buildings as well; the combination of these two effects reinforces the complexity of the upstream flow.

The configuration of multiple rotors enables the acquisition of more fluid energy that obtained with single rotor. In this context, the upstream flow of the downstream rotor bears distinct impression of the wake flow of the front rotor [9]. It is explicit that the wake flow of the Savonius rotor exhibits unevenness and unsteadiness. Therefore, the flow condition faced by the downstream rotor boosts the deviation between practical operation and the basic assumption for rotor design. Regarding the combined rotor with the Savonius rotor being surrounded by three Darrieus rotor blades, the upstream flow of the Savonius rotor is dominated by the rotating wake flows of the lift-type blades $[10,11]$. As the shedding vortex elements enter the flow region covered by the rotation of the Savonius rotor, the transient variation of torque coefficient of the rotor might be affected by twin frequencies, vortex shedding frequency and rotor blade frequency.

As the Savonius rotor rotates, the flow immediately upstream of the rotor is fundamentally the superimposition of the uniform upstream flow and the rotating flows driven by the rotor [12]. This argument is easy to materialize with the conventional Savonius rotor since the cross sections along the rotor axis are identical. While for the Savonius rotor with twisted blades, common techniques are deficient in the presence of complex upstream flow patterns in the presence of the fully three-dimensional velocity. Furthermore, flow principles supporting the optimization the upstream flow and the operation of the Savonius rotor have not established so far [13]. In terms of the flow control, is has only been discussed in vertical lift-type wind rotor in the suppression of the stall phenomenon [14]. Nevertheless, the strategies feasible for the lift-type rotor cannot be transplanted into the drag-type rotor owing to the considerable imparity in rotor operation principles.

Measurement techniques and numerical simulation are prevalent in treating flows near the Savonius rotor. The application of non-intrusive flow velocity techniques such as particle image velocimetry (PIV) and laser Doppler velocimetry (LDV) deepens the understanding of flow characteristics near the Savonius rotor. Nevertheless, for twisted rotor blades, flow measurement still suffers from uncertainties. In contrast, numerical simulation proves to be more reliable. With computational fluid dynamics (CFD) technique, flow details are fully revealed near the Savonius rotor irrespective of the rotor structure or blade shape [15]. A prerequisite for reliable CFD results is the proper specification of upstream flow conditions [16]. Currently, the most intricate and uncertain factor in the application of CFD technique is the turbulence model. Actually, no universally adaptable turbulent model has been invented.

The success of the Savonius rotor is not limited in wind energy utilization. The concept of exploiting the kinetic energy of flowing water is enriched with the introduction of the hydraulic Savonius rotor [17]. In this context, the upstream flow to which the Savonius rotor is exposed involves wavy and oscillating flow structures. Undoubtedly, the density of the medium should be taken into account. This is a fairly new application of the Savonius rotor but has attracted a lot of interest from academic and engineering fields. In addition, the hydraulic Savonius rotor can be used as a velocity measurement tool based on the linear relationship between the velocity of undisturbed upstream flow and rotor rotational speed [18].

The purpose of the present study is to review extensively studies of the upstream flow of the Savonius rotor and to throw light on the flow mechanism underlying upstream flow control. Since no report has been found to offer a specific discussion of the flows upstream of the Savonius rotor, the present study will extract related viewpoints and results from the published literature and compile them into different categories. Furthermore, fairly new patterns of the upstream flow are presented, which is motivated by practical applications of the Savonius rotor. Four types of upstream flows are covered in the present study, namely uniform flow, guided flow, rotor wake flow and oscillating flow. The fluid medium considered is extended from air to water, which means that the Savonius rotor can be used to absorb water energy, which is involved as well in the present study. A global view of 
the development of the upstream flow control methods is enabled with the review. The relationship between the upstream flow and the performance of the Savonius rotor is clarified, which provides a sound support for the improvement of the rotor performance via upstream flow control.

To supplement upstream patterns, numerical simulation and experiments were performed in the present study. For numerical simulation, the numerical scheme adopted in [19] was used here. Flows near the Savonius rotor are assumed as impressible. Therefore, Reynolds averaged Navier-Stokes (RANS) equations, including continuity and momentum equations, are used as governing equations. A turbulent model is required to accomplish the closure of the governing equations. Thus far, the suitability of turbulent model is still a controversial issue [20]. Here, the one-equation Spalart-Allmaras turbulence model is selected. This can properly treat complex flows and the boundary layer effects near the rotor blade [21]. Apart from the numerical scheme, grid deployment plays another important role in the production of valid numerical results [22]. In the present study, for all numerical cases, the equiangle skewness of the grids ranges from 0.2 to 0.28 ; the values of $y+$ range from 27 to 61 . For the interface between rotating and non-rotating domains, the fidelity of data exchange was ensured through adjusting grid density near the interface. The commercial code ANSYS Fluent was employed as the numerical solver.

\section{Fundamental Flow Characteristics of the Savonius Rotor}

The Savonius rotor falls into the drag-type rotor category. The geometry of the conventional Savonius rotor is featured by S-shaped cross sections. Thus far, the rotor geometry has been improved based on the conventional Savonius rotor [23]. For instance, the Savonius rotor with twisted blades has been proved to be more adaptable to wind directions compared to the conventional one. Nevertheless, the principles underlying the rotor design have not established yet. In this case, empirical relationship and judgment are prevalent. Fundamentally, the design of the Savonius rotor conforms to the Betz's elementary momentum theory [24]. The Betz's theory offers a rather ideal explanation of the kinetic energy received by the rotor as air passes through the rotor. The premise for the Betz's theory is that rotor is exposed to uniform upstream flow. With uniform upstream flow, the mass and energy balance associated with the control volume enclosing the Savonius rotor can be described explicitly. Nevertheless, owing to the rotor rotation, both the uniformity and steadiness of the upstream flow cannot be guaranteed. Hitherto, the knowledge about near-rotor flows and fluid pressure over rotor blade surface is far from sufficient. And the latter is directly related to the torque and power output of the Savonius rotor.

A conventional two-bladed Savonius rotor is schematically shown in Figure 1a. As for the returning blade, its rotation is hindered by the upstream flow, and a part of the kinetic energy carried by the upstream flow is not transferred into useful work. Consequently, the energy utilization ratio is undermined. Such a rationale is illustrated by the flow pattern near the Savonius rotor, which was obtained in the present study and is displayed in Figure $1 \mathrm{~b}$. It is seen that the returning blade is exposed to the resistance of the uniform upstream flow. At this moment, the upstream flow near the rotor is obviously influenced by the convex and concave blade surfaces. The initially straight and parallel streamlines featuring the upstream flow are distorted due to the invasion of the rotor surface. The numerical result obtained here is similar to that documented in [25]. Additionally, with such a rotor orientation with respect to the upstream flow, a large torque coefficient is predictable; the contribution of the advancing blade is predominant for the torque production. 


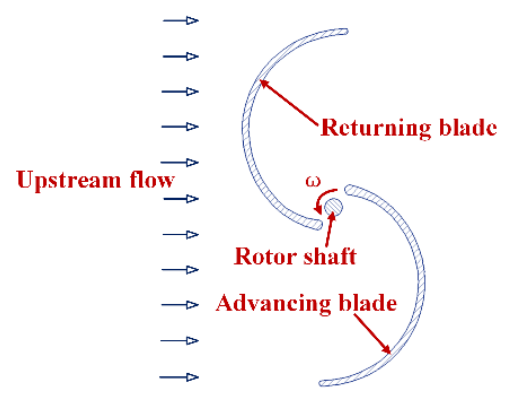

(a)

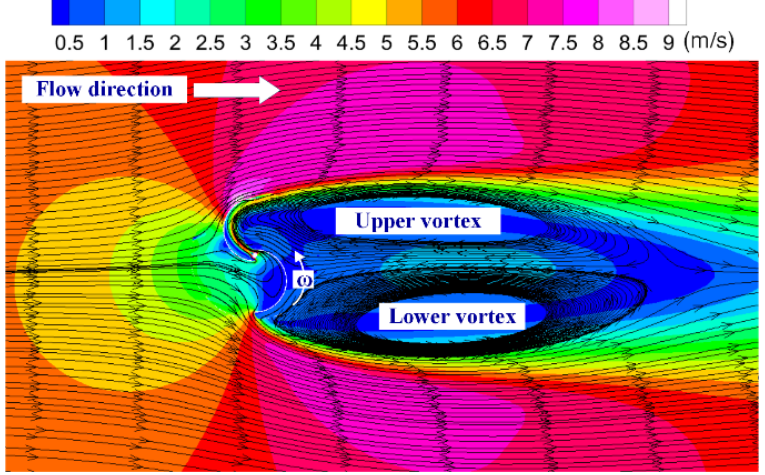

(b)

Figure 1. Conventional Savonius rotor. (a) Schematic view of the rotor; (b) Cross-sectional flow pattern around the rotor.

\section{Upstream Flow Control via Auxiliary Device}

\subsection{Curtains Deployed Upstream of the Rotor}

In view of the flow velocity distribution displayed in Figure 1, it is desirable that the resistance to the counter-clockwise rotation of the returning blade is minimized. This can be accomplished through installing a pair of curtains upstream of the Savonius rotor, which is shown in Figure 2a [26]. Curtains play the role of guiding the upstream flow towards improving the ratio of effective work. Upstream flow patterns obtained in the present study are shown in Figure 2 as well. It is seen that the two curtains guide the upstream flows to impinge the advancing blade, as indicated in Figure $2 \mathrm{~b}$. For the returning blade, the left and right sides are dominated by fairly equivalent pressure distributions, as shown in Figure 2c. Thus the resistance to the rotor delivered through the returning blade is undermined. Moreover, a comparison between Figures $1 b$ and $2 b$ testifies that the wake flow characteristics change considerably with the participation of the curtains. Meanwhile, such an upstream flow control strategy improves the operation performance of the Savonius rotor [27].

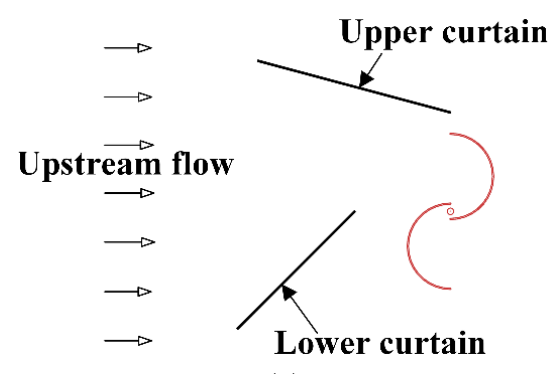

(a)

Figure 2. Cont. 

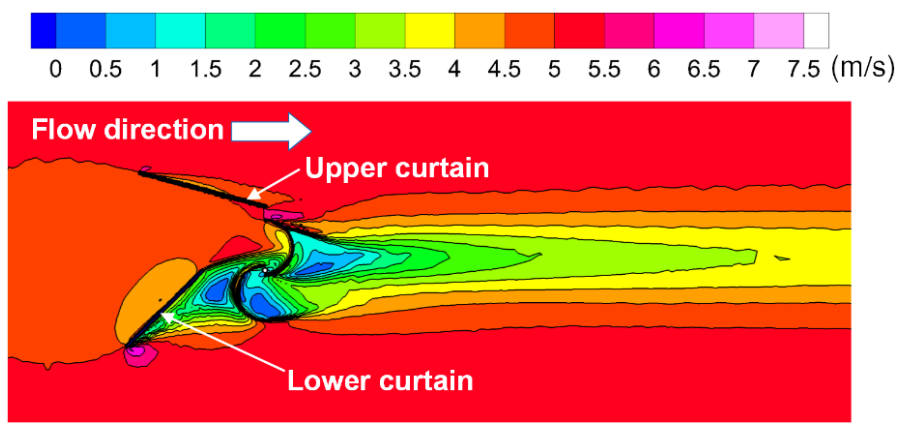

(b)
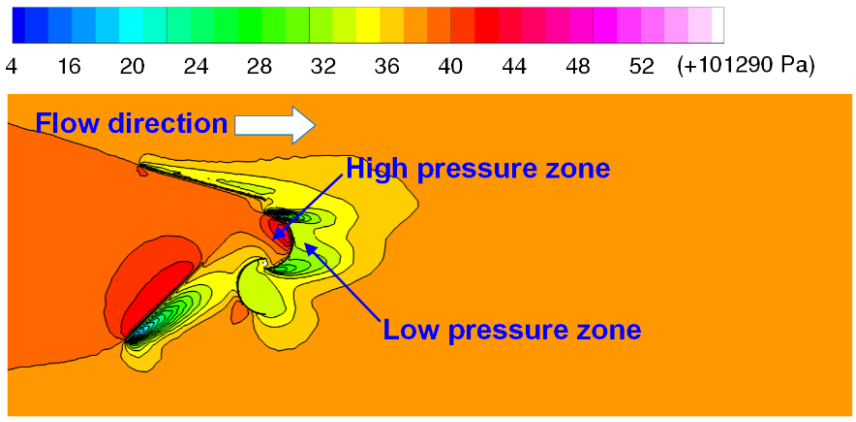

(c)

Figure 2. Cross-sectional flow parameter distributions with upstream curtains. (a) Configuration of upper and lower curtains [26]; (b) Velocity distribution; (c) Static pressure distribution.

The most distinct advantage of numerical simulation is that static pressure can be acquired, which cannot be obtained using existing experimental techniques. In the present study, based on the numerical results associated with the two cases shown in Figures 1 and 2, the static pressure over the rotor blade surface was integrated to calculate the torque exerted on the whole rotor. In Figure 3 , the torque coefficient is plotted as a function of the angle of rotation based on the numerical results. It is explicit that negative torque coefficients are completely eliminated with the deployment of the two curtains, although the maximum torque coefficients of the two cases are nearly equal. This means that energy conversion for the scheme with the curtains is more effective compared to its counterpart. Alternatively, no energy is consumed to hinder the rotation of the rotor.

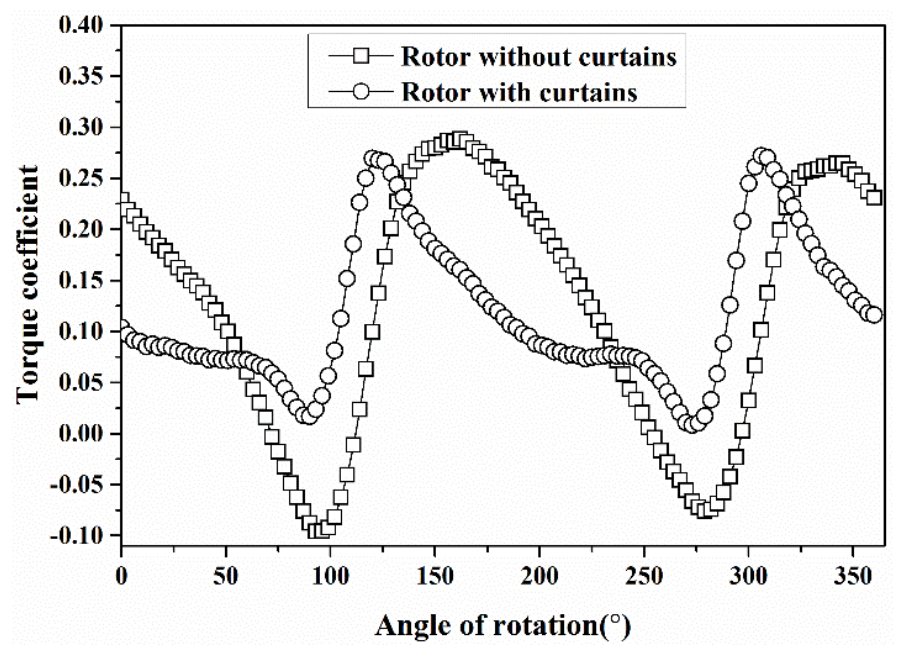

Figure 3. Comparison of torque coefficient between the schemes with and without curtains. 


\subsection{Single Upstream Deflector}

With respect to the situation demonstrated in Figure 2, the lower curtain is apparently more important than the upper curtain since it directly blocks the upstream flow that will otherwise impact on the returning blade. Actually, the attempt of using this curtain exclusively was made in [28], as illustrated in Figure 4a. In this context, curtain, plate and deflector are three names with the same meaning. The flow parameter distributions with such a configuration were simulated in the present study, and the results are displayed in Figure $4 b, c$. It is seen that the flow field gives an impetus to the clockwise rotation of the rotor. Another flow feature in Figure $4 \mathrm{~b}$ is the high-velocity band near the convex side of the returning blade. High velocity is related to the clearance between the returning blade and the stagnation region downstream of the deflector. Therefore, the pressure difference over the two sides of the returning blade also contributes to the anti-clockwise rotation of the rotor, as indicated in Figure 4c. Further study is necessitated to optimize the relative position between the deflector and the rotor, the deflector setting angle and the deflector length.

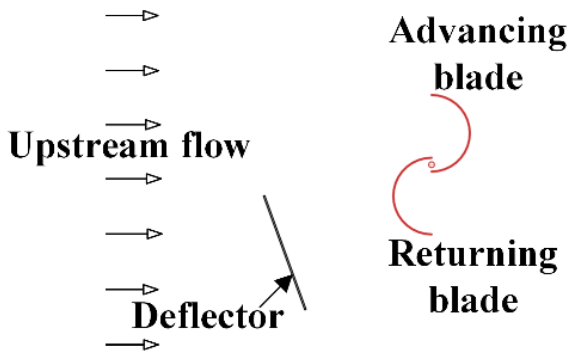

(a)

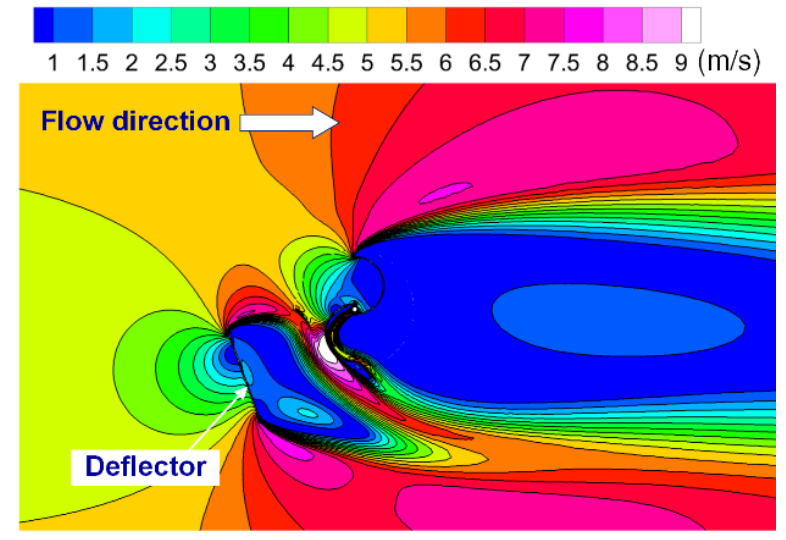

(b)

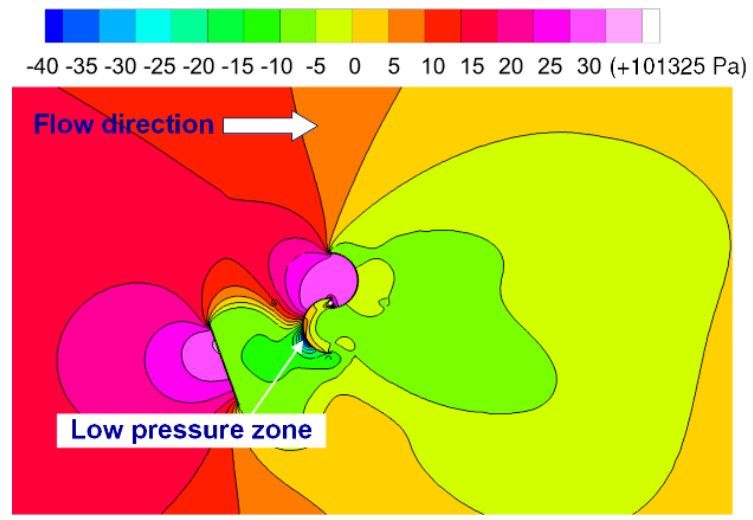

(c)

Figure 4. Cross-sectional flow parameter distributions with single deflector. (a) Single deflector scheme [28]; (b) Velocity distribution; (c) Static pressure distribution. 


\subsection{Scheme of Shield Plates}

A shield plate was mounted upstream of the Savonius rotor as the rotor was used to absorb the kinetic energy of water [29]. With such a scheme, an increase in the power coefficient was proved. Essentially, the influence of adverse pressure gradients can be averted via the deployment of the shield plate, which serves as the most important reason of the improvement of rotor performance. A more flexible scheme of shield plates recorded in [30] is schematically shown in Figure 5a. It is seen that two curved plates constituted a short duct within which the Savonius rotor was installed. In the present study, numerical simulation for such a scheme was performed and corresponding flow parameter distributions are shown in Figure 5b,c. It is noticeable that the duct bounded by the two plates is not parallel with the upstream flow. In the duct, the advancing blade is subjected to advantageous pressure difference, while the resistance to the returning blade is mitigated with the jet-like flow structure near the tip of the returning blade, as shown in Figure 5b. Moreover, for the returning blade, pressure at the concave side is higher than that at the convex side, as indicated in Figure 5c; this facilitates the counter-clockwise rotation of the rotor as well. Furthermore, it has been proved in [31] that the combination of the Savonius rotor and shield plates promotes the rotor performance at all rotor rotation angles.

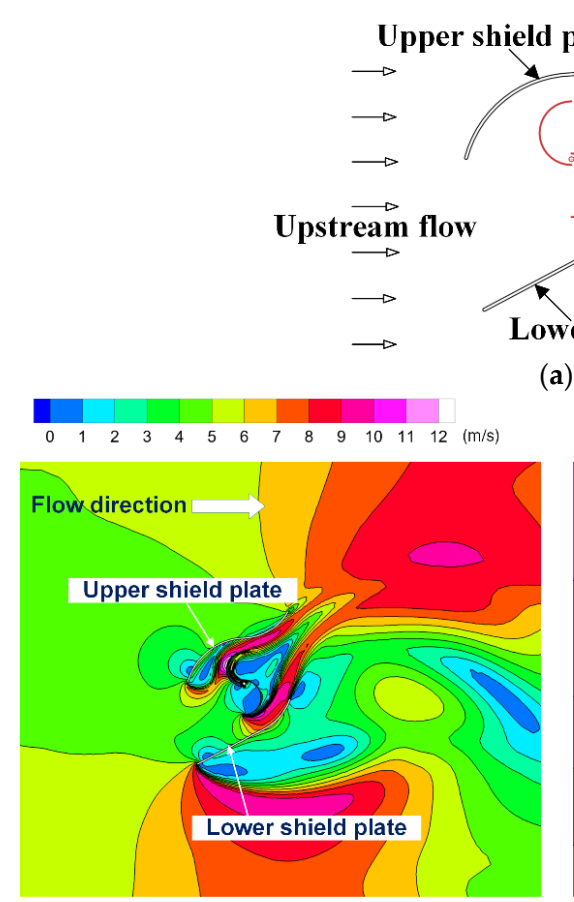

(b)

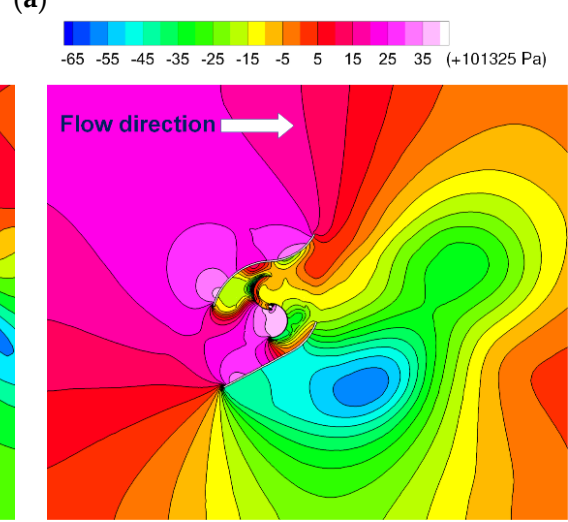

(c)

Figure 5. Scheme of curved shield plates. (a) Scheme featured by two curved plates [30]; (b) Velocity distribution; (c) Static pressure distribution.

An even more intricate configuration of the auxiliary plates with various geometrical shapes is exhibited in Figure 6 [32], where the flow characteristics are illustrated as well. The invention of such a combined structure with three plates is based inevitably on flow analysis. As implied in Figure 6, a part of the upstream flow is controlled by curtains. Another part of the upstream flow is guided into a flow passage along a twisted route. A branch of the flows in this passage will impinge the concave side of the returning blade. In this context, both blades are associated with favorable pressure distributions. Nevertheless, it can be inferred from the flows trapped in the complex flow passage lower than the returning blade that losses of fluid energy are remarkable. There is sufficient space for the optimization of the plate geometry and the relative position between the rotor and the plates. 


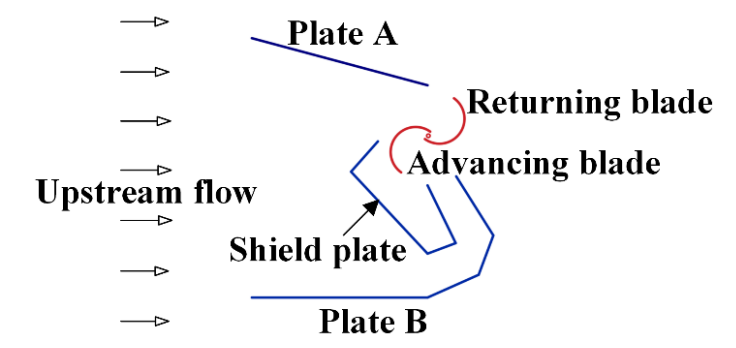

(a)

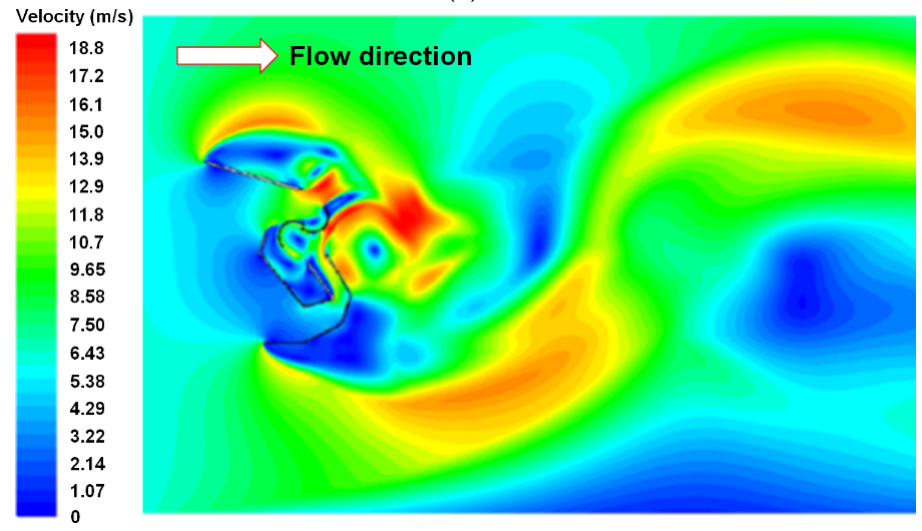

(b)

Figure 6. Configuration of three auxiliary plates [32]. (a) Schematic view of complex auxiliary plates; (b) Velocity distribution.

\subsection{Plates Mounted both Upstream and Downstream}

The rotor is a rotating component while the adjacent plates are stationary; it is perceivable that the flow patterns near the Savonius rotor are determined by the two significantly different solid boundaries jointly [33]. In some cases, the configuration of the plates is asymmetric with respect to the rotor axis, which enhances the non-uniformity of flow parameter distributions near the rotor. As shown in Figure 7a, the Savonius rotor is surrounded by four plate; two are straight type and the other two include both straight and curved segments [34]. Here, numerical simulation was performed to describe flow patterns near the rotor and the results are displayed in Figure 7b,c. Near the rotor blades, flow deflection, gap flow, impinging flow and vortex flow are evidently displayed in Figure $7 \mathrm{~b}$. In this case, the motivation of deploying the plates upstream of the rotor is to avoid the resistance to the returning blade. It is noteworthy that with Plate C, a gap flow is formed between this plate and the convex side of the advancing blade. Such a gap flow impinges the concave side of the returning blade, giving an impetus to the counter-clockwise rotation of the rotor. Plate B is deployed downstream of the rotor, with this plate, as the rotor rotates, there will always be a duct that assumes the similar function as that implied in Figure 5. The pressure distribution near the rotor blades is shown in Figure 7c. The thrust behind the counter-clockwise rotation of the rotor is remarkable, as attributes to the pressure difference over the two sides of each rotor blade.

From another viewpoints, the introduction of multiple plates leads to an increase in the contact area between the medium and the rotor [35]. Therefore, the medium is required to experience a long energy-transformation process before it is finally relieved from the restriction of the rotor and plates. Such a practice is in good accordance with general principles of fluid energy utilization. A practice intended to accomplish the similar effect is to deploy end plates for the Savonius rotor. Such a practice is particularly suitable for the twisted Savonius rotor, which is characterized by near-rotor flows along the axial direction [36]. 


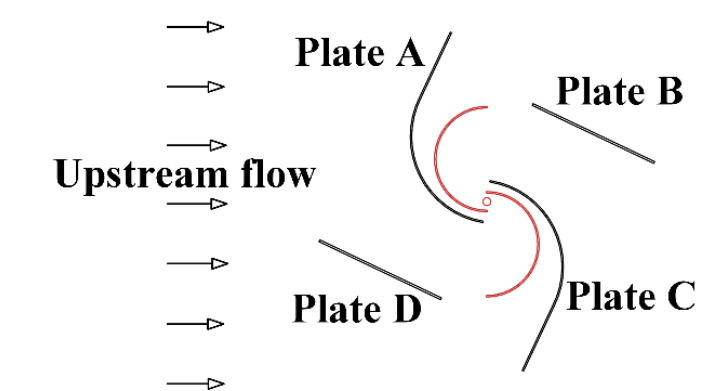

(a)

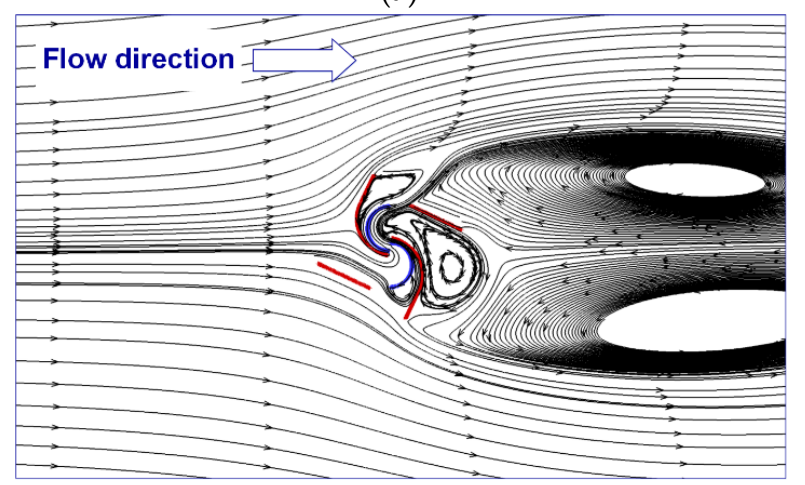

(b)

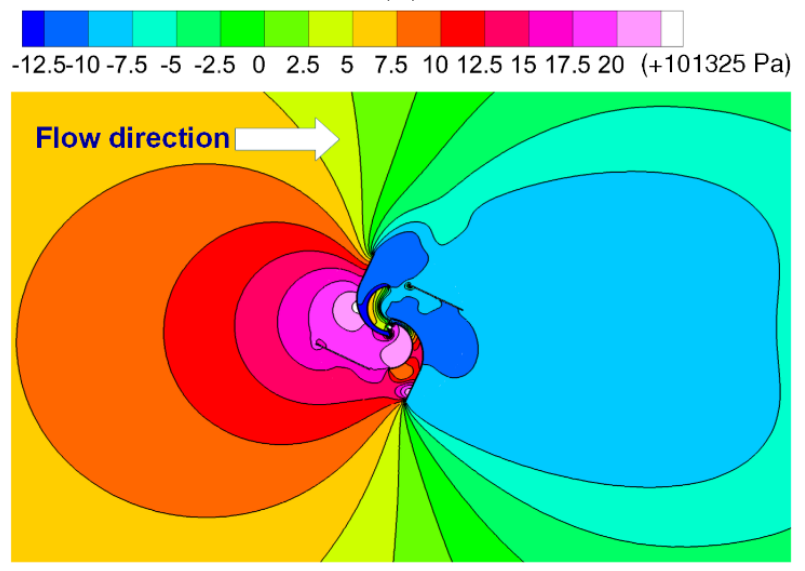

(c)

Figure 7. Scheme of asymmetrically arranged auxiliary plates. (a) Sketch of the multiple-plate scheme [34]; (b) Streamlines; (c) Pressure distribution.

\subsection{Rotor Performance Improvement with Upstream Flow Control}

Apart from the torque coefficient described in Figure 3, the power coefficient can be used to assess the performance of the Savonius rotor as well. Regarding practical applications, these two coefficients are more detectable than flow parameters. Here, a comparison of rotor performance between Savonius rotors with and without auxiliary device was implemented. The results are illustrated in Table 1, where $c_{t}$ denotes the torque coefficient, $c_{p}$ denotes the power coefficient and TSR represents the tip-speed ratio. ' $w /$ ' represents the condition with auxiliary device; while 'w/o' represents the condition without auxiliary device. The data listed in Table 1 were obtained experimentally or numerically. The experimental approach for acquiring rotor performance data is straightforward, while for the numerically obtained torque and power coefficients, they were calculated through integrating static pressure over the rotor blade surface. 
Table 1. Comparison of rotor performance between schemes with and without auxiliary device.

\begin{tabular}{ccccccc}
\hline Reference & Device & $\mathbf{c}_{t}(\mathbf{w} / \mathbf{o})$ & $\mathbf{c}_{\boldsymbol{t}}(\mathbf{w} /)$ & $\mathbf{c}_{\boldsymbol{p}}(\mathbf{w} / \mathbf{o})$ & $\mathbf{c}_{\boldsymbol{p}}(\mathbf{w} /)$ & TSR \\
\hline$[26]$ & curtain & - & - & 0.16 & 0.38 & 0.4 \\
{$[28]$} & obstacle & 0.23 & 0.33 & 0.17 & 0.25 & 0.8 \\
{$[29]$} & shield plate & - & - & 0.24 & 0.48 & 1.4 \\
{$[30]$} & curtain & 0.31 & 0.37 & 0.24 & 0.29 & 0.8 \\
{$[32]$} & deflector & 0.20 & 0.53 & 0.19 & 0.52 & 1.0 \\
{$[35]$} & baffles & - & - & 0.15 & 0.32 & 0.6 \\
{$[37]$} & deflector & 0.15 & 0.23 & 0.13 & 0.21 & 0.9 \\
{$[38]$} & obstacle & 0.23 & 0.33 & 0.18 & 0.26 & 0.8 \\
\hline
\end{tabular}

Apparently, the rotor performance is universally improved by using the auxiliary device. As for the case documented in [32], the power coefficient with the deflector is more than twice of the original value. A considerable improvement of rotor performance is also found in the case studied in [29], which is featured by the introduction of the shield plate. In this connection, apart from the auxiliary device, there are other factors that influence rotor performance, such as rotor blade size, blade shape, blade surface roughness and tip-speed ratio, any of them can outweigh the others in the contribution to the torque or energy coefficient. A full consideration of all these factors is impractical in most cases. Furthermore, flow characteristic is a reflection of the synthetic effect of these factors, thus it is difficult to establish an optimization scheme towards improving the quality of the flow based on the identification of the effect of any individual factor.

\section{Configuration of Multiple Rotors}

Fundamentally, more energy can be extracted from the nature with multiple Savonius rotors. Nevertheless, inappropriate rotor arrangement might results in low rotor performance and even rotor operation instability. In this case, the interaction among neighboring rotors is significant. A two-rotor scheme proposed in [39] is shown in Figure 8. It is seen that the gap between two rotors is small, and both rotors suffer from the influence of the gap flow. With the synchronous rotation of the two rotors, the gap flow will take time-dependent form and affects the flow velocity distribution in the circular rotor rotation region periodically.

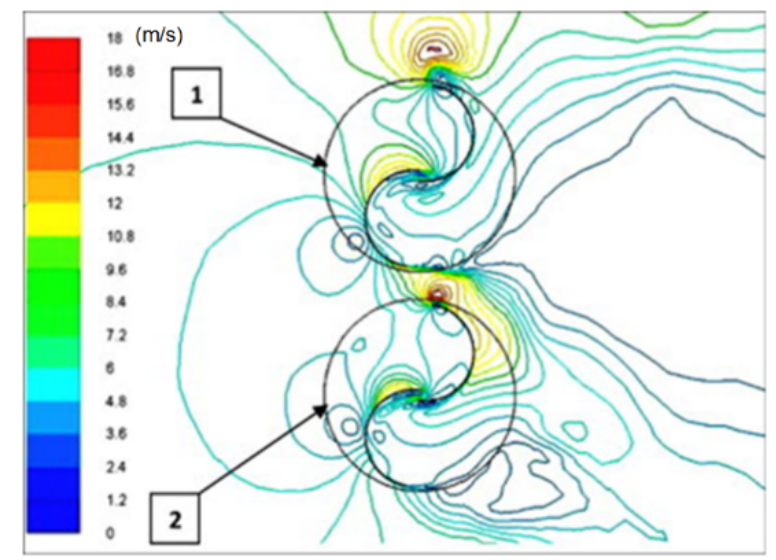

Figure 8. The two-rotor scheme and corresponding flow pattern [39].

In the present study, another two-rotor scheme was suggested and the flow was numerically treated; the flow velocity distribution is illustrated in Figure 9. The two rotors are identical and the streamwise distance between the rotation axes of the two rotors is three times the rotor diameter. It can be judged from local flow velocity distribution that the downstream rotor suffers from the influence of the wake flow of the upstream rotor. It is evident that the flow patterns near the advancing blades of 
the two rotors are different. Thus far, the upstream flow faced by the downstream rotor can only be visualized using numerical strategies and the shortage of experimental techniques is appreciable in the presence of the rotor-rotor interaction.

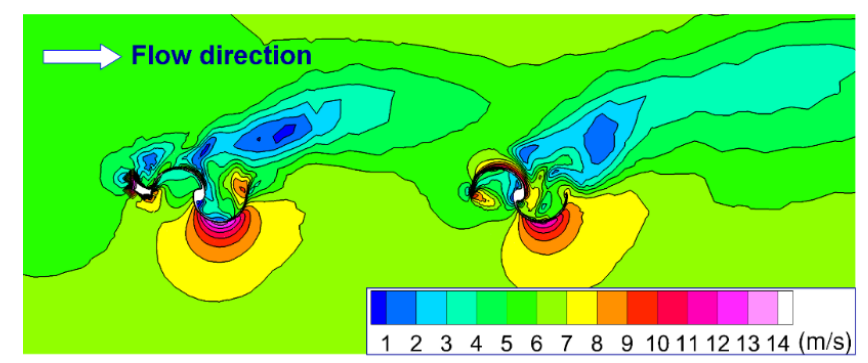

Figure 9. Flow velocity distribution near the upstream and downstream rotors.

To investigate the effect of the inter-rotor distance on the upstream flow of the downstream rotor, the streamwise distance between two rotors shown in Figure 9 is enlarged to five times the rotor diameter. Numerical simulation was performed for this configuration as well. Velocity distributions over three horizontal lines evenly distributed between the two rotors are constructed and plotted in Figure 10. The non-dimensional abscissas of 0 and 1 represent the two rotation region edges, respectively, as marked in Figure 10. The imparity between the three velocity curves is remarkable near the upstream rotor, as is related to the strong effect of the wake flow. Then the three curves experience a fairly smooth stage, which signifies that the effect of the upstream rotor decays in streamwise direction. Near the downstream rotor, obvious deviation between the three curves arises again, and this is determined by the boundary effect and rotation of the downstream rotor. As for the optimum inter-rotor distance, it is related to the tip-speed ratio and rotor geometry. Frequently, turbulent fluctuations in this unique upstream flow should be considered, enabling an evaluation of the reliability of the rotor and the operation stability of the whole unit.

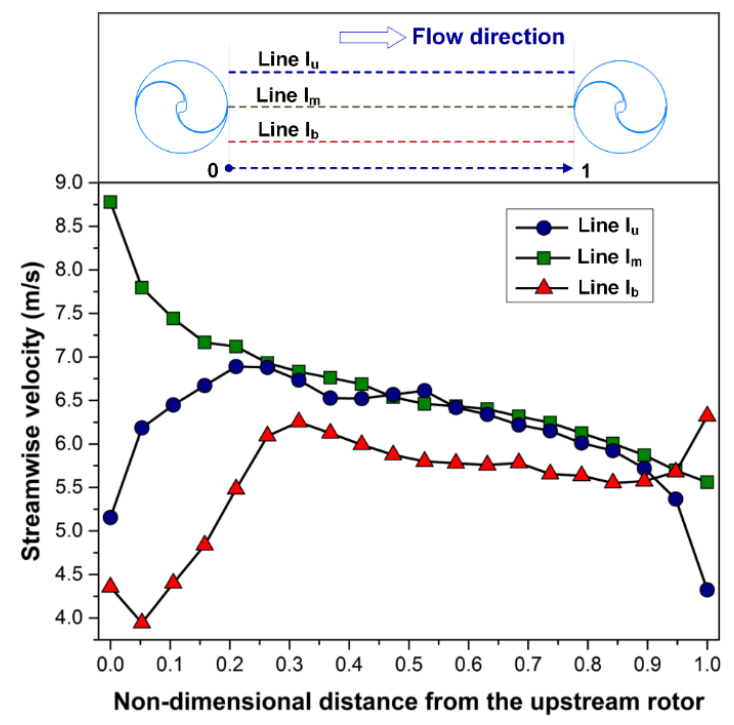

Figure 10. Velocity distributions between the upstream and downstream rotors.

As three or more rotors operates in the same field, the rotor-rotor interaction deserves a consideration in both streamwise and transverse directions [40]. A representative is the triple-rotor scheme, as shown in Figure 11. The three rotors, namely Rotors A, B and C, are positioned with fairly uneven inter-rotor space. For the two downstream rotors, Rotors $B$ and $C$, the upstream flows are 
different. Meanwhile, the interaction between the two downstream rotors is possible. In [41], velocity distributions near the three rotors are presented, as displayed in Figure 11b. It is recognizable that Rotor B is exempted from the influence of the wake-type upstream flow, but the upstream flow of Rotor C is partially impressed by the wake flow of Rotor A. It should be pointed out that the rotation directions of Rotors $\mathrm{A}$ and $\mathrm{C}$ are opposite. Thus vortices in the wake flow of Rotor A are beneficial for the clockwise rotation of Rotor C, as can be envisaged based upon wake flow features of Rotor A, which rotates in anti-clockwise direction.

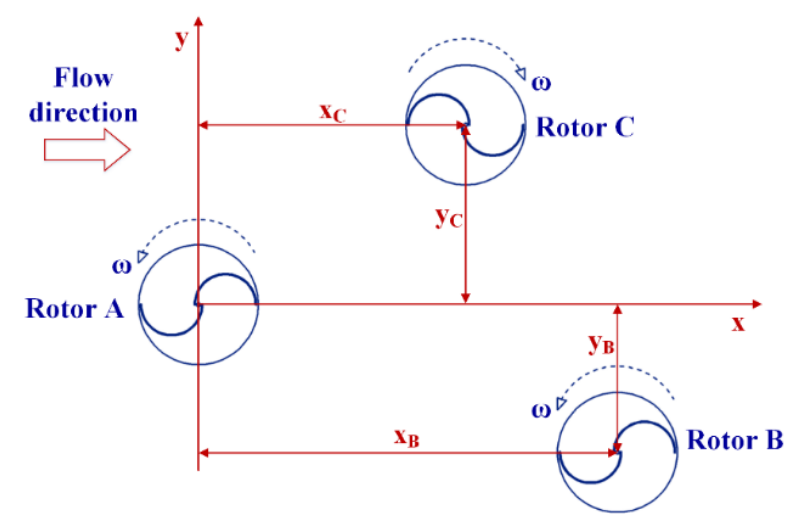

(a)

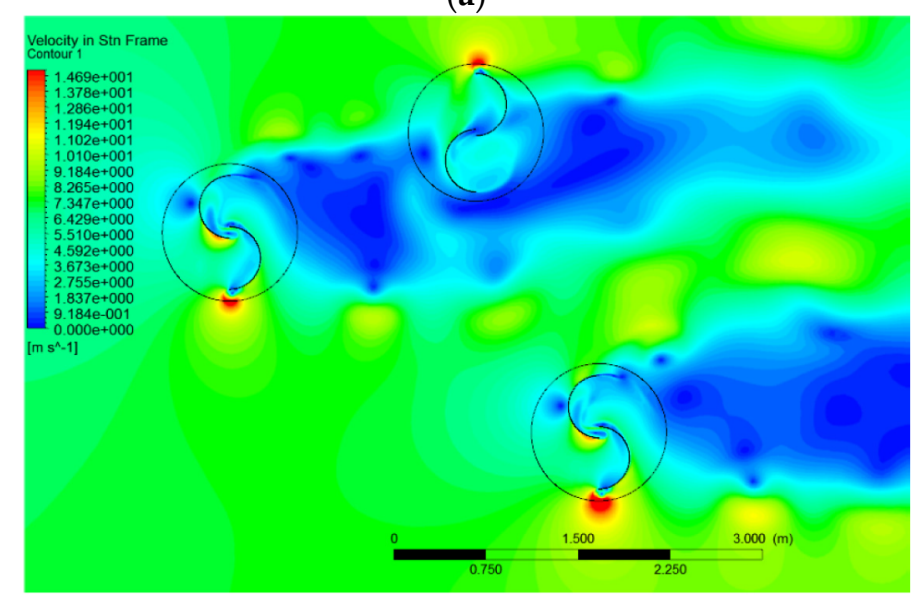

(b)

Figure 11. Scheme of three rotors and flow characteristic [41]. (a) Schematic view of the three-rotor configuration; (b) Velocity distribution near the three rotors.

Provided that the Savonius rotors employed in the wind farm are equipped with three-dimensional curved blades, the upstream flow situation encountered by the downstream rotor will bear clear spatial non-uniformity along the direction parallel to the rotor axis. In the present study, three-dimension flows for such a configuration were simulated, the result is illustrated in Figure 12. 


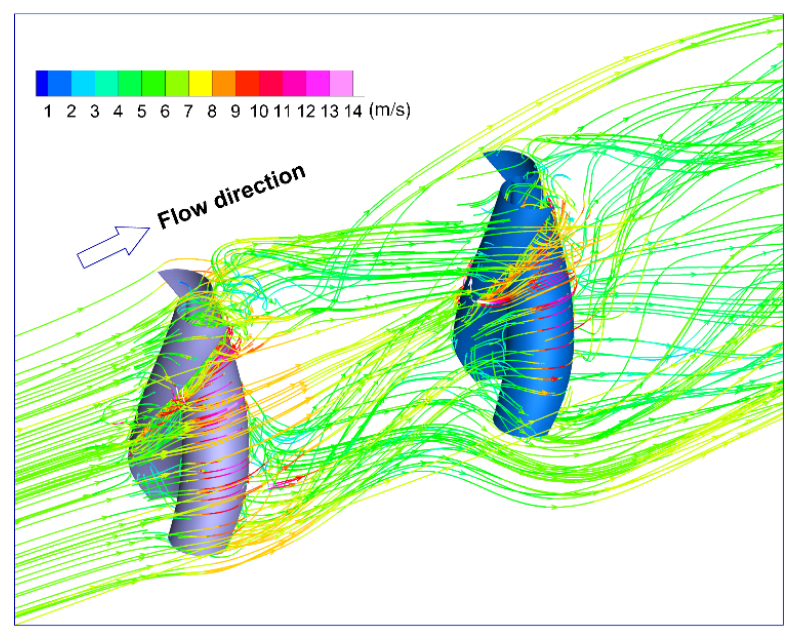

Figure 12. Three-dimensional streamlines near two Savonius rotors with curved blades.

As is evidently clear, the streamlines between the two rotors are considerably distorted. In comparison, streamlines near the downstream rotor are more irregular, which can be traced to the spatially non-uniform wake of the upstream rotor. A special feature of the Savonius rotor with curved blades is the upstream flow will be trapped into the space between the two blades, then travels along the blade passage, finally detaches from the confinement of the rotor. Therefore, the upstream control strategies involving auxiliary curtains or plates cannot be applied directly in the curved-bladed Savonius rotor.

Orderly arranged rotors, along with the flow velocity distribution, are displayed in Figure 13. The configuration was reported in [42], and the numerical work was performed in the present study to describe flow parameter distributions. From a global view, the near-rotor flow patterns are analogous for the three rotors. The orientation of Rotor B is seemingly more beneficial relative to that of the other two rotors. This can be inferred from the remarkable large-velocity area near the convex side of the advancing blade. Therefore, the wake flow can be an effective upstream flow for rotor clusters on the premise that the relative positions of the rotors are properly devised. In addition, the bulk flow direction serves as another critical factor affecting flow characteristics of the multiple-rotor combination. Variation in the bulk flow direction causes an alteration of the relative streamwise position between the upstream and downstream rotors. Consequently, the performance of the downstream rotor undergoes a change accordingly. Thus far, the largest number of Savonius rotors constituting a wind farm was reported in [43], where 27 Savonius wind turbines were considered. In this case, a full consideration of the interaction between neighboring rotors at the initial stage of the rotor arrangement design is necessitated.

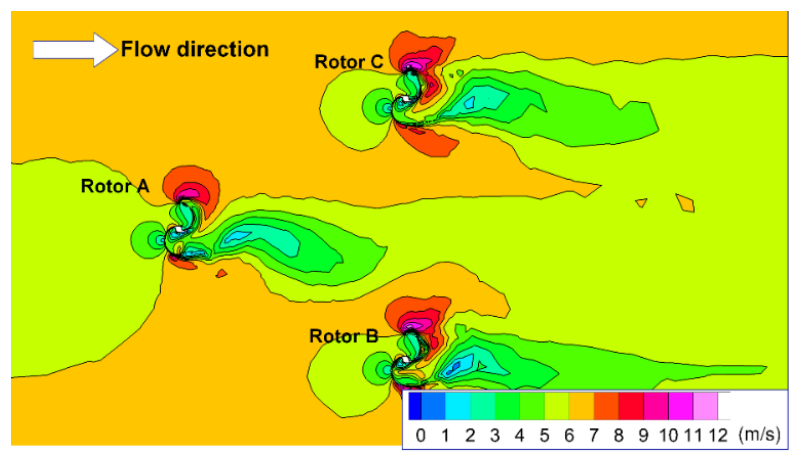

Figure 13. Three-rotor scheme and corresponding velocity distributions. 


\section{Upstream Flow in Tunnels and Pipes}

\subsection{Upstream Flow in Tunnels}

In some occasions, the validation of the upstream flow control strategy can be implemented via experiments. Thus far, the wind tunnel and water tunnel are two prevalent experimental facilities for testing the Savonius rotor [44,45]. Nevertheless, the overwhelming majority of published studies focus on the operation performance of the rotor instead of flow details, although flow visualization or measurement can be realized with these facilities. For the wind tunnel, it can operate in open or closed manner, and the tested Savonius rotor can be installed in the test segment or exposed to ambient air. While for the water tunnel, the rotor has to be fixed in the test segment. To attain high upstream flow velocity, the cross-sectional area of the test segment is minimized. Thus the influence of the solid wall of the test segment turns to be non-negligible [46]. From the perspective of fluid dynamics, the upstream flow of the tested rotor is dominated by the boundary layer effect and deviates from phenomenally the assumption of infinite flow domain [47].

In the present study, a Savonius rotor was installed in the test segment of a water tunnel, as shown in Figure 14. The side walls of the test segment are made of plexiglass, enabling non-intrusive measurement or visualization of flows in the test section. Generally, two-dimensional Savonius rotor models are suitable for flow measurement. With the twisted Savonius rotor, the velocity component in transverse direction would disturb the data acquisition on the monitored plane.

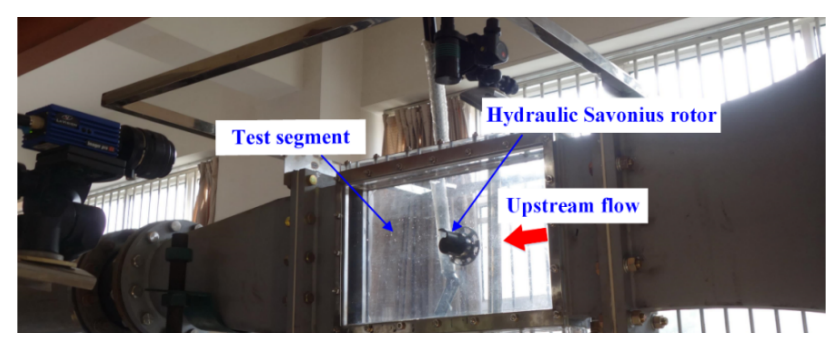

Figure 14. Savonius rotor installed in the test section of a water tunnel.

Here, the flow upstream of the Savonius rotor was measured using PIV and cross-sectional velocity distributions with various velocity magnitudes are plotted in Figure 15. It is seen that the velocity distribution over the middle part of the monitored cross section is rather smooth, as is common for various upstream flow velocities. Near the two side walls of the test segment, velocity drops sharply towards the wall. Therefore, it is desirable that the tested Savonius rotor is mounted in the uniform portion of the upstream flow.

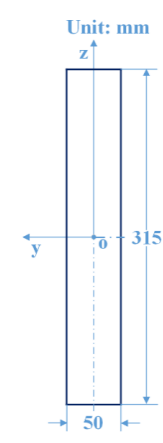

(a)

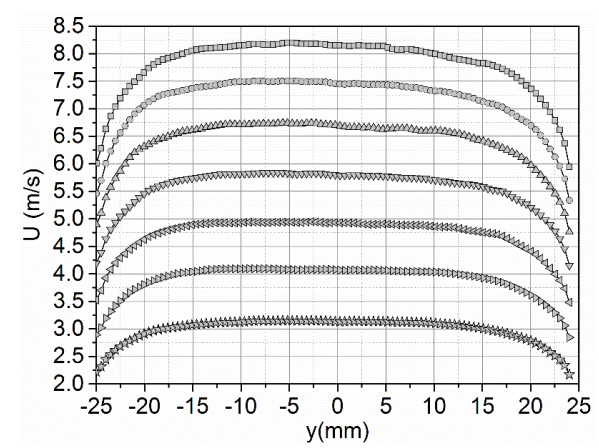

(b)

Figure 15. Cross-sectional upstream flow velocity distributions in the test section of a water tunnel: (a) cross-section dimensions of the test segment; (b) velocity distributions at different upstream velocity magnitudes. 
The test section geometry imposes a significant influence on upstream flow. It is reported in [48] that a convergent flow passage is deployed upstream of a Savonius rotor. In [49], the geometry of the flow passages upstream of the Savonius rotor is specifically designed. One of the major functions of the test section is to provide uniform and stable upstream flow for the Savonius rotor. Except flow velocity distributions shown in Figure 15, turbulent intensity is another index of evaluating upstream flow quality in the water or wind tunnel. This is related to the fact that turbulent fluctuations are important in determining the performance of the rotor [50]. In addition, with existing experimental facilities and apparatus, static pressure distribution near the rotor cannot be obtained [51].

\subsection{Upstream Flow in the Pipe}

Pipe flows are ubiquitous and time-averaged velocity distributions in the pipe have been well understood. The Savonius rotor could extract the kinetic energy from flowing water in pipes [52]. Under such a condition, velocity profiles upstream of the Savonius rotor can be given. Nevertheless, with the participation of the Savonius rotor, the characteristic resistance curve of the pipe system is altered, which will affect the operation of other components of the system. Furthermore, installing a small rotor inside a large pipe is desirable. In one aspect, the effects of the rotor to the whole system are relieved; in the other aspect, the effects of the pipe wall on the upstream flow would be attenuated as well.

A scheme with a Savonius rotor installed in a pipe was reported in [53]. The uniqueness of such a scheme lies in that a semi-circular shield was used to shield half of the rotor, as shown in Figure 16a. In the present study, numerical simulation was carried out to describe flow patterns associated with such a scheme. The flow velocity distribution obtained numerically is displayed in Figure 16b. With such a baffle, the negative influence of the upstream flow on the anticlockwise rotation of the rotor is alleviated considerably. Meanwhile, a high-velocity zone is seen on the upper side of the baffle. This is due to the minimizing of the flow area between the pipe wall and the convex surface of the baffle. The rotor is equipped with five blades, enlarging the contact area between the rotor and fluid. The role played by the shield shown in Figure 16 is similar to that assumed by the curtains and the deflector, which are indicated in Figures 2 and 4. Similar concept has been advised in [54], where proof was presented to support the improvement of the rotor performance due to partially blocked upstream flow as the bulk flow was confined in a duct.

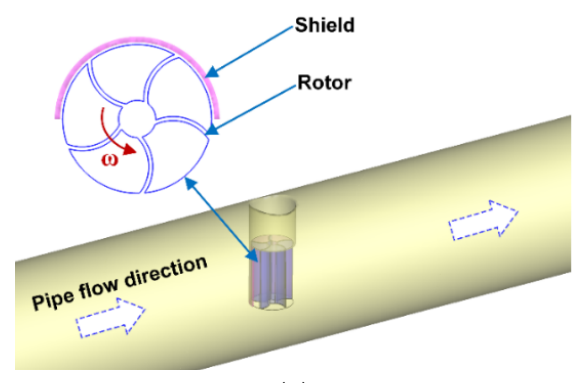

(a)

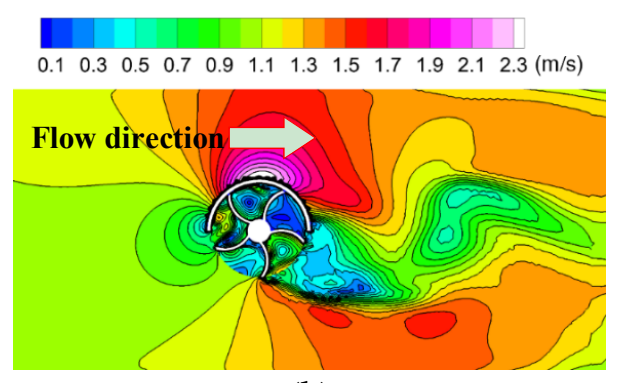

(b)

Figure 16. Savonius rotor and the semi-circular shield in the pipe: (a) schematic view [53]; (b) cross-sectional velocity distribution.

Flows in the pipe or duct are relatively coherent; therefore the energy concentration is high, which improves the kinetic energy utilization rate of the Savonius rotor. It has been proved in [55] that the Savonius rotor in the test section of a tunnel possesses a high torque coefficient. In practice, the investigation of flow characteristics for the Savonius rotor operating under such conditions has been conducted overwhelmingly using numerical simulation. Regarding the setting of boundary conditions, the wall effects must be given a full consideration [56,57]. Any deviation from the physical truth might give rise to spurious results. Additionally, it is necessary that the power-law 
upstream velocity profile is accurately imposed on the computational domain as a velocity inlet boundary condition.

During the operation of the rotor, the rotation angle of the rotor varies periodically, the flow between the rotor and the wall of the tunnel or pipe exhibits significantly different patterns [58]. As the Savonius rotor is placed in a duct with streamwise convergent geometry, along with the unique feature of the flow field, the maximum fluid load exerted on the rotor is high relative to that obtained under common operation conditions [59]. Furthermore, as the rotational speed of the rotor increases, the oscillation of the wake flow downstream of the rotor is intensified. Consequently, the vibration of the pipe or the duct due to flow excitation deserves a careful inspection.

\section{Wavy Upstream Flow}

The exploitation of the tidal energy is facilitated with the application of the Savonius rotor, as has been substantiated in recent years [60]. Meanwhile, water waves provide an advantageous operation condition for the so-called hydraulic Savonius rotor. The Savonius rotor outweighs other rotors in the magnitude of power output, as in inseparable from its large blade surface area. Both experimental and numerical techniques have been attempted to imitate the tidal energy utilization environment. Therefore, the understanding of such a fairly new application of the Savonius rotor has been constantly deepened. The method of producing wavy upstream flow is crucial for investigating the performance or flow characteristics of the Savonius rotor used to absorb the wave energy. In [61], a wave generator is deployed upstream of the Savonius rotor, while in [62], a wave flume assumes the similar role. Alternatively, numerical simulation can produce the oscillating upstream flow [63]. In comparison, numerical techniques are flexible in controlling the amplitude and the frequency of the virtually generated wave.

In the present study, numerical techniques were used to produce the wavy upstream flow for a Savonius rotor. Based on the numerical results, five consecutive upstream flow patterns were constructed, as displayed in Figure 17. Here, $T_{0}$ represents a reference moment. It is seen that the upstream flows at different moments exhibit various patterns. The effects of the wavy upstream flow structure are spread over a large portion of the flow surrounding the Savonius rotor. The Savonius rotor is exposed to an exceedingly unstable environment. It is inferable that the time-dependent power output of the rotor is influenced by the wavy characteristics as well. In this context, the design of such a Savonius rotor should consider multiple factors besides flow parameters, such as blade material, structural strength, even the risk of vibration should be taken into account in some cases [64]. Provided that the rotor is permitted to float in the liquid medium, the correlation between rotor displacement, rotor rotation and wavy upstream flow necessities a synthetic investigation. Regarding the kinetic energy utilization in off-shore regions, the hydraulic Savonius rotor is sometimes immersed partially in water during the operation. In this case, the upstream flow of the rotor is subject to the influence of not only flow structures beneath the free surface but also the wavy free surface itself [65]. Therefore the numerical treatment must employ multiphase numerical models such as the volume of fluid (VOF) model in conjunction with turbulent models to depict the unsteady fluctuations fostered in the upstream flow. At present, the understanding of such a subject is still inadequate.

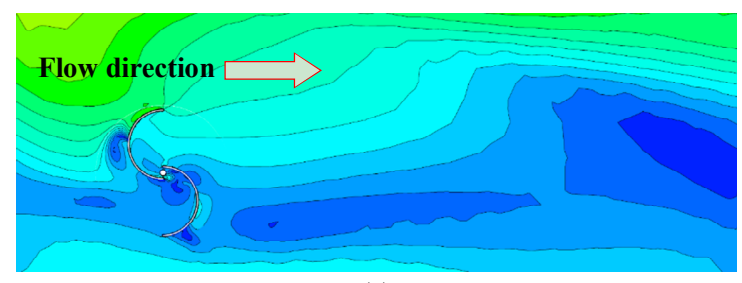

(a)

Figure 17. Cont. 


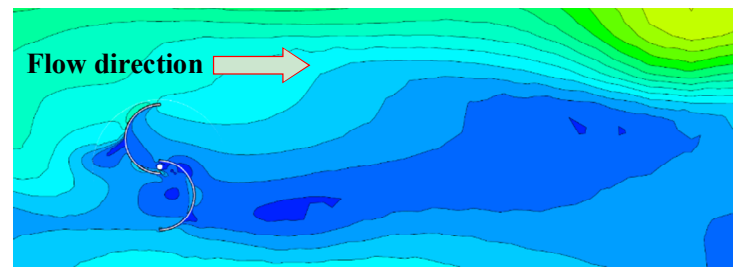

(b)

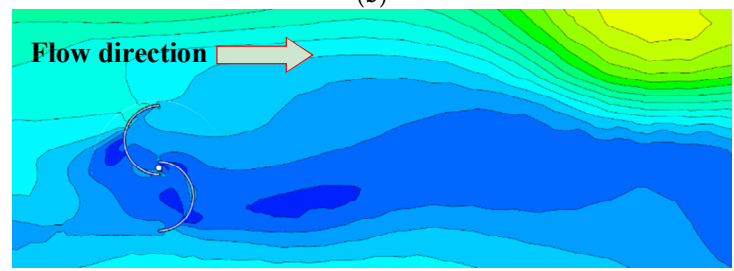

(c)

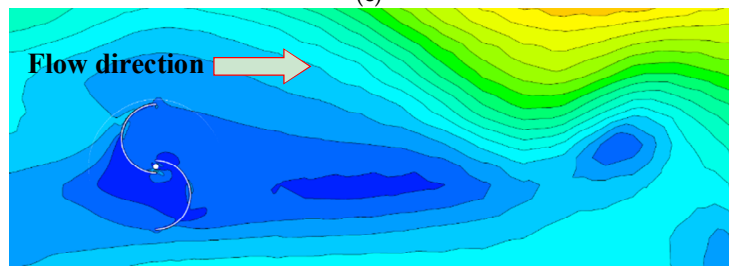

(d)

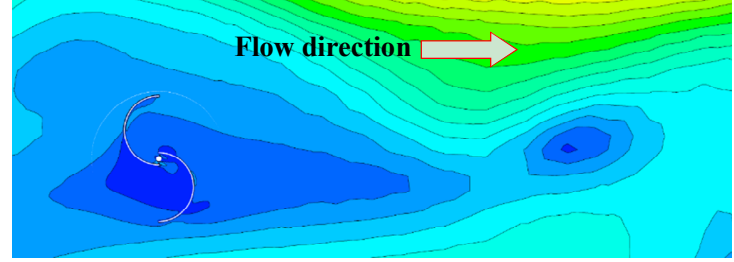

(e)

$\begin{array}{lllllllllllllllllllll}0.0 & 0.1 & 0.2 & 0.3 & 0.4 & 0.5 & 0.6 & 0.7 & 0.8 & 0.9 & 1.1 & 1.2 & 1.3 & 1.4 & 1.5 & 1.6 & 1.7 & 1.8 & 1.9 & 2.0\left[\mathrm{~m} \mathrm{~s}^{\wedge}-1\right]\end{array}$

Figure 17. Time-dependent velocity distributions in wavy flows near a Savonius rotor. (a) $T_{0} ;(\mathbf{b}) T_{0}+0.25 \mathrm{~s}$; (c) $T_{0}+0.5 \mathrm{~s} ;$ (d) $T_{0}+0.75 \mathrm{~s} ;(\mathbf{e}) T_{0}+1.0 \mathrm{~s}$.

\section{Concluding Remarks}

Various upstream flows for the Savonius rotor are presented and analyzed in the present study. Flow characteristics are described and the factors influencing the flow patterns are explained, which deepens the understanding of the principles underlying the upstream flow control methods. Major conclusions of the study are as follows.

(1) Diverse upstream flow patterns have been witnessed in the studies of the Savonius rotor. Although there is no established research branch for the upstream flow control, the importance of the upstream flow for the rotor performance is acknowledged. Flow characteristics serve as a bridge between the upstream flow and the rotor performance, which is substantiated in this review.

(2) Apart from relevant studies documented in published literature, an extension of the upstream flow is attained. Experimental and numerical techniques are employed to explain the effects of the upstream flow. Both air and water are used to drive the rotation of the Savonius rotor. Pipe flow and wave are demonstrated as distinct upstream flows for the application of the Savonius rotor.

(3) The validation of the upstream flow control strategies is accomplished via numerical simulation; more specifically, the output torque and power coefficient of the Savonius rotor can be calculated based on numerically obtained static pressure distributions over rotor blades. This facilitates the development of more effective measures of upstream flow control for the Savonius rotor. 
Author Contributions: C.K. conceived of the main idea and wrote the paper. W.O. analyzed the data of the rotors investigated. C.P. performed the experiments. Z.Z. contributed to the numerical simulation.

Acknowledgments: The authors are grateful to the Six Talent Peaks Project in Jiangsu Province of China (Grant No. 2015-ZBZZ-018) and the College Industrialization Project of Jiangsu province of China (Grant No. JHB2011-37). The authors also thank the Computer Center of Jiangsu University for providing the authors with the high-performance computer cluster.

Conflicts of Interest: The authors declare no conflict of interest.

\section{References}

1. Tummala, A.; Velamati, R.K.; Sinha, D.K.; Indraja, V.; Krishna, V.H. A review on small scale wind turbines. Renew. Sustain. Energy Rev. 2016, 56, 1351-1371. [CrossRef]

2. Maldonado, R.D.; Huerta, E.; Corona, J.E.; Ceh, O.; León-Castillo, A.I.; Gómez-Acosta, M.P.; Mendoza-Andrade, E. Design, simulation and construction of a Savonius wind rotor for subsidized houses in Mexico. Energy Procedia 2014, 57, 691-697. [CrossRef]

3. Gupta, A.K. Efficient wind energy conversion: Evolution to modern design. J. Energy Resour. Technol. 2015, 137, 051201. [CrossRef]

4. Afungchui, D.; Kamoun, B.; Helali, A. Vortical structures in the wake of the Savonius wind turbine by the discrete vortex method. Renew. Energy 2014, 69, 174-179. [CrossRef]

5. Altan, B.D.; At1lgan, M. Study on increasing the performance of Savonius wind rotors. J. Mech. Sci. Technol. 2012, 26, 1493-1499. [CrossRef]

6. Ali, A.; Golde, S.; Alam, F.; Moria, H. Experimental and computational study of a micro vertical axis wind turbine. Procedia Eng. 2012, 49, 254-262. [CrossRef]

7. Goh, S.C.; Boopathy, S.R.; Krishnaswami, C.; Schlüter, J.U. Tow testing of Savonius wind turbine above a bluff body complemented by CFD simulation. Renew. Energy 2016, 87, 332-345. [CrossRef]

8. ELMokadem, A.A.; Megahed, N.A.; Noaman, D.S. Systematic framework for the efficient integration of wind technologies into buildings. Front. Archit. Res. 2016, 5, 1-14. [CrossRef]

9. Baz, A.M.; Mahmoud, N.A.; Hamed, A.M.; Youssef, K.M. Optimization of two and three rotor Savonius wind turbine. In Proceedings of the ASME Turbo Expo 2015: Turbine Technical Conference and Exposition, Montréal, QC, Canada, 15-19 June 2015.

10. Irwan, Y.M.; Daut, I.; Safwati, I.; Irwanto, M.; Gomesh, N.; Fitra, M. A new technique of photovoltaic/wind hybrid system in Perlis. Energy Procedia 2013, 36, 492-501. [CrossRef]

11. Bhuyan, S.; Biswas, A. Investigations on self-starting and performance characteristics of simple $\mathrm{H}$ and hybrid H-Savonius vertical axis wind rotors. Energy Convers. Manag. 2014, 87, 859-867. [CrossRef]

12. Nasef, M.H.; El-Askary, W.A.; AbdEL-hamid, A.A.; Gad, H.E. Evaluation of Savonius rotor performance: Static and dynamic studies. J. Wind Eng. Ind. Aerodyn. 2013, 123, 1-11. [CrossRef]

13. Wong, K.H.; Chong, W.T.; Sukiman, N.L.; Poh, S.C.; Shiah, Y.C.; Wang, C.T. Performance enhancements on vertical axis wind turbines using flow augmentation systems: A review. Renew. Sustain. Energy Rev. 2017, 73, 904-921. [CrossRef]

14. Sasson, B.; Greenblatt, D. Effect of leadings-edge slot blowing of a vertical axis wind turbine. AIAA J. 2011, 49, 1932-1942. [CrossRef]

15. Kang, C.; Liu, H.; Yang, X. Review of fluid dynamics aspects of Savonius-rotor-based vertical-axis wind rotors. Renew. Sustain. Energy Rev. 2014, 33, 499-508. [CrossRef]

16. Park, S.H.; Park, J.H.; Park, J.C.; Lee, E.T. The performance of small wind power generation systems on super high-rise buildings. Int. J. Steel Struct. 2014, 14, 489-499. [CrossRef]

17. Kumar, A.; Saini, R.P. Performance parameters of Savonius type hydrokinetic turbine-A Review. Renew. Sustain. Energy Rev. 2016, 64, 289-310. [CrossRef]

18. Anderson, F.E. The shot-sensor method of measuring currents in shallow estuarine waters. Estuaries 1990, 13, 250-257. [CrossRef]

19. Sharma, S.; Sharma, R.K. Performance improvement of Savonius rotor using multiple quarter bladesA CFD investigation. Energy Convers. Manag. 2016, 127, 43-54. [CrossRef]

20. Roy, S.; Saha, U.K. Review on the numerical investigations into the design and development of Savonius wind rotors. Renew. Sustain. Energy Rev. 2013, 24, 73-83. [CrossRef] 
21. Kang, C.; Yang, X.; Wang, Y. Turbulent flow characteristics and dynamics response of a vertical-axis spiral rotor. Energies 2013, 6, 2741-2758. [CrossRef]

22. Lam, H.F.; Peng, H.Y. Study of wake characteristics of a vertical axis wind turbine by two-and threedimensional computational fluid dynamics simulations. Renew. Energy 2016, 90, 386-398. [CrossRef]

23. Chen, L.; Chen, J.; Zhang, Z. Review of the Savonius rotor's blade profile and its performance. J. Renew. Sustain. Energy 2018, 10, 013306. [CrossRef]

24. Hau, E. Wind Turbines, Fundamentals, Technologies, Application, Economics, 2nd ed.; Springer: Berlin/Heidelberg, Germany, 2006.

25. Goodarzi, M.; Keimanesh, R. Numerical analysis on overall performance of Savonius turbines adjacent to a natural draft cooling tower. Energy Convers. Manag. 2015, 99, 41-49. [CrossRef]

26. Altan, B.D.; Atılgan, M.; Özdamar, A. An experimental study on improvement of a Savonius rotor performance with curtaining. Exp. Therm. Fluid Sci. 2008, 32, 1673-1678. [CrossRef]

27. Altan, B.D.; Atılgan, M. An experimental and numerical study on the improvement of the performance of Savonius wind rotor. Energy Convers. Manag. 2008, 49, 3425-3432. [CrossRef]

28. Mohamed, M.H.; Janiga, G.; Pap, E.; Thévenin, D. Optimal blade shape of a modified Savonius turbine using an obstacle shielding the returning blade. Energy Convers. Manag. 2011, 52, 236-242. [CrossRef]

29. Iio, S.; Katayama, Y.; Uchiyama, F.; Sato, E.; Ikeda, T. Influence of setting condition on characteristics of Savonius hydraulic turbine with a shield plate. J. Therm. Sci. 2011, 20, 224-228. [CrossRef]

30. Tartuferi, M.; D'Alessandro, V.; Montelpare, S.; Ricci, R. Enhancement of Savonius wind rotor aerodynamic performance: A computational study of new blade shapes and curtain systems. Energy 2015, 79, 371-384. [CrossRef]

31. Montelpare, S.; D’Alessandro, V.; Zoppi, A.; Ricci, R. Experimental study on a modified Savonius wind rotor for street lighting systems. Analysis of external appendages and elements. Energy 2018, 144, 146-158. [CrossRef]

32. El-Askary, W.A.; Nasef, M.H.; Abdel-Hamid, A.A.; Gad, H.E. Harvesting wind energy for improving performance of Savonius rotor. J. Wind Eng. Ind. Aerodyn. 2015, 139, 8-15. [CrossRef]

33. Tesch, K.; Kludzinska, K.; Doerffer, P. Investigation of the aerodynamics of an innovative vertical-axis wind turbine. Flow Turbul. Combust. 2015, 95, 739-754. [CrossRef]

34. Wahyudi, B.; Soeparman, S.; Hoeijmakers, H.W. Optimization design of Savonius diffuser blade with moving deflector for hydrokinetıc cross flow turbıne rotor. Energy Procedia 2015, 68, 244-253. [CrossRef]

35. Yao, Y.X.; Tang, Z.P.; Wang, X.W. Design based on a parametric analysis of a drag driven VAWT with a tower cowling. J. Wind Eng. Ind. Aerodyn. 2013, 116, 32-39. [CrossRef]

36. Jeon, K.S.; Jeong, J.I.; Pan, J.K.; Ryu, K.W. Effects of end plates with various shapes and sizes on helical Savonius wind turbines. Renew. Energy 2015, 79, 167-176. [CrossRef]

37. Golecha, K.; Eldho, T.I.; Prabhu, S.V. Influence of the deflector plate on the performance of modified Savonius water turbine. Appl. Energy 2011, 88, 3207-3217. [CrossRef]

38. Mohamed, M.H.; Janiga, G.; Pap, E.; Thévenin, D. Optimization of Savonius turbines using an obstacle shielding the returning blade. Renew. Energy 2010, 35, 2618-2626. [CrossRef]

39. Shaheen, M.; El-Sayed, M.; Abdallah, S. Numerical study of two-bucket Savonius wind turbine cluster. J. Wind Eng. Ind. Aerodyn. 2015, 137, 78-89. [CrossRef]

40. Shigetomi, A.; Murai, Y.; Tasaka, Y.; Takeda, Y. Interactive flow field around two Savonius turbines. Renew. Energy 2011, 36, 536-545. [CrossRef]

41. Zhang, B.; Song, B.; Mao, Z.; Tian, W. A novel wake energy reuse method to optimize the layout for Savonius-type vertical axis wind turbines. Energy 2017, 121, 314-355. [CrossRef]

42. El-Baz, A.R.; Youssef, K.; Mohamed, M.H. Innovative improvement of a drag wind turbine performance. Renew. Energy 2016, 86, 89-98. [CrossRef]

43. Shaheen, M.; Abdallah, S. Development of efficient vertical axis wind turbine clustered farms. Renew. Sustain. Energy Rev. 2016, 63, 237-244. [CrossRef]

44. Ricci, R.; Romagnoli, R.; Montelpare, S.; Vitali, D. Experimental study on a Savonius wind rotor for street lighting systems. Appl. Energy 2016, 161, 143-152. [CrossRef]

45. Driss, Z.; Mlayeh, O.; Driss, S.; Maaloul, M.; Abid, M.S. Study of the incidence angle effect on the aerodynamic structure characteristics of an incurved Savonius wind rotor placed in a wind tunnel. Energy 2016, 113, 894-908. [CrossRef] 
46. Frikha, S.; Driss, Z.; Ayadi, E.; Masmoudi, Z.; Abid, M.S. Numerical and experimental characterization of multi-stage Savonius rotors. Energy 2016, 114, 382-404. [CrossRef]

47. Al-Faruk, A.; Sharifian, A. Geometrical optimization of a swirling Savonius wind turbine using an open jet wind tunnel. Alex. Eng. J. 2016, 55, 2055-2064. [CrossRef]

48. Promdee, C.; Photong, C. Effects of Wind Angles and Wind Speeds on Voltage Generation of Savonius Wind Turbine with Double Wind Tunnels. Procedia Comput. Sci. 2016, 86, 401-404. [CrossRef]

49. Goundar, J.N.; Prasad, D.; Ahmed, M.R. Design and performance testing of a ducted Savonius turbine for marine current energy extraction. In Proceedings of the ASME 2013 International Mechanical Engineering Congress and Exposition, San Diego, CA, USA, 15-21 November 2013.

50. Liu, H.; Jin, Y.; Tobin, N.; Chamorro, L.P. Towards uncovering the structure of power fluctuation of wind farms. Phys. Rev. E 2017, 96, 063117. [CrossRef] [PubMed]

51. Murai, Y.; Nakada, T.; Suzuki, T.; Yamamoto, F. Particle tracking velocimetry applied to estimate the pressure field around a Savonius turbine. Meas. Sci. Technol. 2007, 18, 2491-2503. [CrossRef]

52. Chen, J.; Yang, H.X.; Liu, C.P.; Lau, C.H.; Lo, M. A novel vertical axis water turbine for power generation from water pipelines. Energy 2013, 54, 184-193. [CrossRef]

53. Altan, B.D. The numerical simulation of the performances of water rotors used in pipelines with the water baffle plates. J. Mech. Sci. Technol. 2014, 28, 4555-4562. [CrossRef]

54. Al-Kayiem, H.H.; Ming, G.J. Experimental investigation of S-rotors in open and bounded flows, World Academy of Science. Eng. Technol. 2011, 60, 144-149.

55. Torresi, M.; Fortunato, B.; Pascazio, G.; Camporeale, S.M. CFD analysis of a Savonius rotor in a confined test section and in open field. In Proceedings of the ASME Turbo Expo, Vancouver, BC, Canada, 6-10 June 2011.

56. D'Alessandro, V.; Montelpare, S.; Ricci, R.; Secchiaroli, A. Unsteady Aerodynamics of a Savonius wind rotor: A new computational approach for the simulation of energy performance. Energy 2010, 35, 3349-3363. [CrossRef]

57. Zhao, Z.; Zheng, Y.; Xu, X.; Liu, W.; Zhou, D. Optimum design configuration of helical Savonius rotor via numerical study. In Proceedings of the ASME 2009 Fluids Engineering Division Summer Meeting, Vail, CO, USA, 2-6 August 2009.

58. Ross, I.; Altman, A. Wind tunnel blockage corrections: Review and application to Savonius vertical-axis wind turbines. J. Wind Eng. Ind. Aerodyn. 2011, 99, 523-538. [CrossRef]

59. Kumar, D.; Sarkar, S. Numerical investigation of hydraulic load and stress induced in Savonius hydrokinetic turbine with the effects of augmentation techniques through fluid-structure interaction analysis. Energy 2016, 116, 609-618. [CrossRef]

60. Bikas, G.S.; Ramesh, H.; Vijaykumar, H. Study on performance of Savonius rotor type wave energy converter used in conjunction conventional rubble mound breakwater. Ocean. Eng. 2014, 89, 62-68. [CrossRef]

61. Patel, S.K.; Ram, K.; Ahmed, M.R.; Lee, Y.H. Performance studies on an oscillating water column employing a Savonius rotor. Sci. China Technol. Sci. 2011, 54, 1674-1679. [CrossRef]

62. Tutar, M.; Veci, I. Performance analysis of a horizontal axis 3-bladed Savonius type wave turbine in an experimental wave flume (EWF). Renew. Energy 2016, 86, 8-25. [CrossRef]

63. Zullah, M.A.; Prasad, D.; Ahmed, M.R.; Lee, Y.H. Performance analysis of a wave energy converter using numerical simulation technique. Sci. China Technol. Sci. 2010, 53, 13-18. [CrossRef]

64. Kumar, A.; Saini, R.P. Performance analysis of a Savonius hydrokinetic turbine having twisted blades. Renew. Energy 2017, 108, 502-522. [CrossRef]

65. Akimoto, H.; Tanaka, K.; Kim, Y.Y. Drag-type cross-flow water turbine for capturing energy from the orbital fluid motion in ocean wave. Renew. Energy 2015, 76, 196-203. [CrossRef]

(C) 2018 by the authors. Licensee MDPI, Basel, Switzerland. This article is an open access article distributed under the terms and conditions of the Creative Commons Attribution (CC BY) license (http:/ / creativecommons.org/licenses/by/4.0/). 